\title{
Proposal of new-type charge system by using contact-type positioning free charger and expendable electrode
}

\author{
Daisuke Tanaka $^{\mathrm{a}}$, Yuki Misaki ${ }^{\mathrm{a}}$, Yang Shiyuan ${ }^{\mathrm{a}}$, Makoto Miyauchi $^{\mathrm{b}}$ \\ ${ }^{a}$ Kyushu Institute of Technology, 1-1 Sensui-cho Tobata-ku Kitakyuusyuu-shi Fukuoka-ken, 804-8550, Japan \\ ${ }^{\mathrm{b}}$ Kitakyushu College of Technology, 5-20-1 Shii, Kokuraminami-ku Kitakyuusyuu, 802-0985 Japan \\ *o349416d@mail.kyutech.jp
}

\begin{abstract}
Since Japan is becoming aging society, many kinds of autonomous robots are expected as labor instead of human being. Their power source is battery because they are required the wide movable area. Therefore the high efficiency charging system will be more important equipment than now. The major styles are "wireless charge type" and "automatic charge type". The first type is not suitable for high power charge. The other type is better than first one but it is important whether the battery is charged correctly or not. If the new type system exists, which can charge just only touching the robots on its certain area without regard to position, these problems would not only solve but also be applied for temporary charge terminal which can use in the contaminated area by radiation or disaster area.

In this study, as one of the method to solve these, we will show the principle of contact-type charger which can use without regard to position and how to design of expendable electrodes.
\end{abstract}

Keywords: positioning-free, contact-type, electrode design

\section{Introduction}

Recently, the number of elderly is increasing rapidly and the amount of nursing home for the elderly is also increasing accordingly. Therefore, instead of the helper, many kinds of autonomous robots are expected as labor to take care of them in such facilities in the near future. However, robots' power consumption is so high because of using a lot of motors that supply method of the electric power to robots efficiently is very significant. If they can be supplied the power automatically, this problem might be solved immediately.

The current major power-supply style is the following three types: "wired style", "battery charging style" and "change style". Each style has some advantages and disadvantages. For example, in wired style, one of the advantages is that robots can use larger amount of electric power than other style and one of the disadvantages is that robots are limited moving area because of power supply cable. In this case, considering management performance and costs, we focused on "battery charging system", especially, "contact-type" because this system can deal with large amount of current than "wireless-type". Therefore we and decided to develop the positioning free contact-type charger. However, when the robots' battery needs to be charged, it is essential operation to judge whether battery's electrode position is correct or not. From these reasons, it is more profitable to charge the battery without specific operation. In addition to that, if the new type electrode exists, which can extend freely under the certain conditions, it will be more convenient and easier to apply or installation.

In this study, we clarify that the principle of contact-type charger which can charge the battery without positioning and the design of electrode which is able to expand the charge area without the increment of electrical components.

\section{Contact-type charger which can charge the battery without positioning}

Many kinds of automatic battery charging systems are already developed. Compared with normal type charger, the probability of breakdown is so high due to mechanical parts that it is important to decrease the probability. Recently, 
wireless chargers have been developed frequently. Most of them don't have mechanical parts but it has other problems that are lower power transmission efficiency, unsuitable for high power charge and longer charge time.

From the above, we developed the automatic contact-type battery charging system. The main characteristics of this system are as follows;

- The circuit is simple and the scale is small.

- We can use it only putting the battery on.

- This can charge without positioning

- Various type batteries can use it.

This system includes three phases; detection phase, switching phase and charging phase.

\subsection{Detection phase}

A flow chart of detection phase is shown in Fig.1. and a

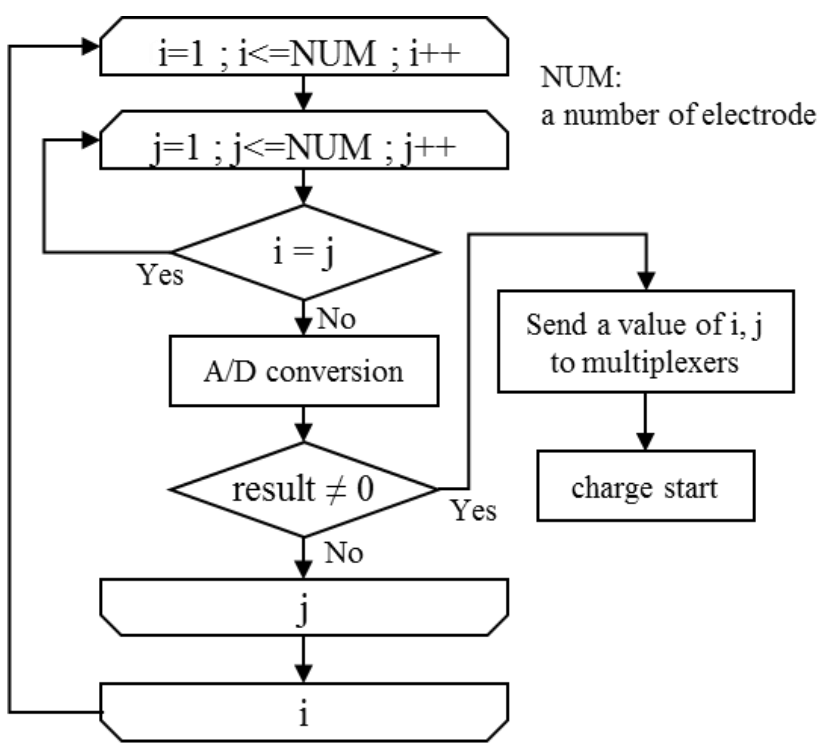

Fig.1. A flow chart of detection phase

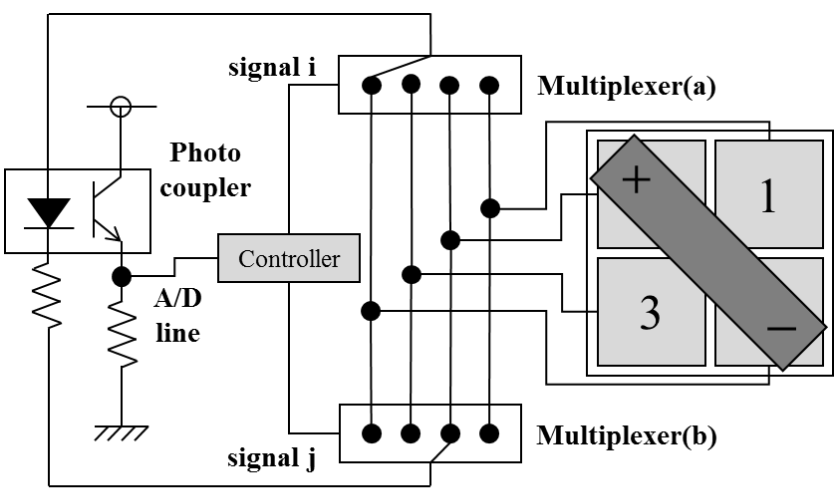

Fig.2. a schematic of detection circuit

schematic of detection circuit is shown in Fig.2. Multiplexers' terminals are connected with not only each other but also same number square electrode in Fig. 2.

The processes of detection phase are as follows;

(1) The controller select the electrodes combination patterns that might happen when the battery is put on electrodes, for example, 1-2, 2-4 and so on.

(2) The controller sends the combination to multiplexers. If the combination is $4-2$, the value " 4 " is sent to the multiplexer(a), " 2 " is sent to the multiplexer(b).

(3) Each multiplexer connect the sent number's terminal with each common terminal. As shown in Fig.2., the multiplexer(a) is connected with No.4 electrode and multiplexer(b) is connected with No. 2 electrode.

(4) After connecting multiplexers with electrodes, the controller starts $\mathrm{A} / \mathrm{D}$ conversion to measure the voltage. In this step, if the photo coupler is connected with the battery through the multiplexers and electrodes correctly, the photo-transistor is turned on and the voltage drop appears. Because of this, A/D conversion result is not zero. On the other hand, if the photo coupler is connected incorrectly, the voltage drop doesn't appear and the $\mathrm{A} / \mathrm{D}$ conversion result is zero.

By using these differences, the controller can detect not only the touching position but also the battery's polarity.

If battery is put like Fig.2., photo-transistor is not turned on but if the combination is " $2-4$ " it is turned on because the current flows.

This charger doesn't change the detection phase to the switching phase and continues to try the combination until the battery is put on it. Thanks to this characteristic, when the other conductor such as metal is put on this, the detection phase is never moved to the charging phase.

\subsection{Switching phase}

A schematic of switching circuit is shown in Fig.3. Fig.3.(a) shows the unconnected state and Fig.3.(b) shows the correct-connected state. The circuit structure of multiplexer is same as decoder, so we utilize multiplexers as decoder to simplify the circuit in this phase. In addition to that, we used MOSFET as a switches because it can deal with larger amount of current than bipolar transistor.

The processes of switching phase are as follows;

(1) After detecting the touching position and battery's polarity, the controller keeps the electrodes combination and multiplexers' common terminal 
are connected with Vcc through the relay to utilize them as decoder to control MOSFETs. (The relay is omitted in Fig.3.)

(2) By keeping the combination values, as shown in Fig.3.(b), the same number's MOSFETs are turned on by decoders.

(3) If the appropriate MOSFETs are turned on, the closed circuit from charge control IC to the battery is composed through the MOSFET.

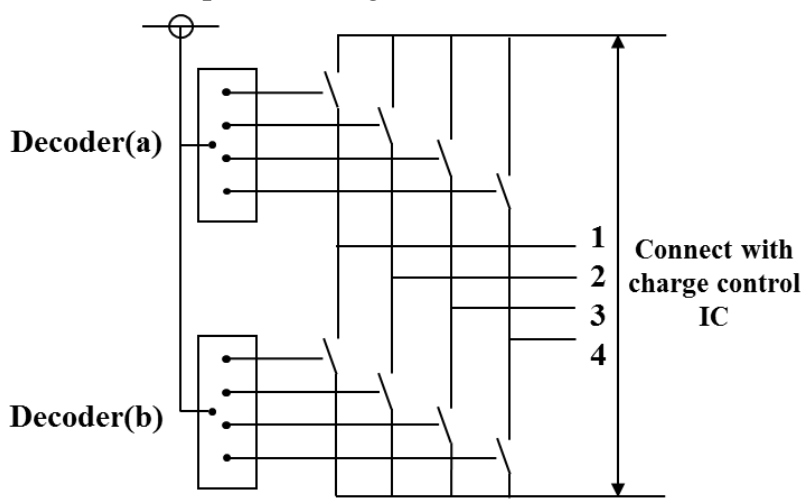

(a)

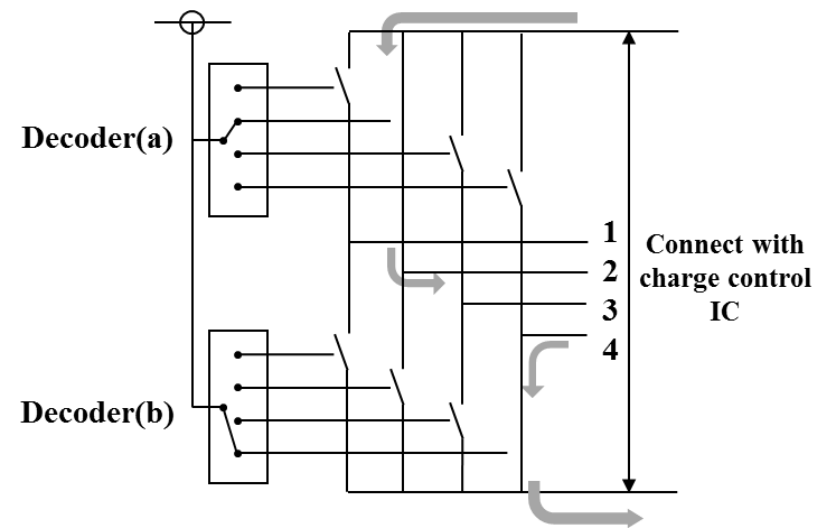

(b)

Fig.3. A schematic of switching circuit

\subsection{Charging phase}

We used the specific charge control IC because it is sensitive to overcharge to charge the battery, especially Li-ion type. The specific charge control IC enables to control the current and voltage strictly.

If this IC is connected with the switching circuit, the battery is charged by constant current (CC) mode at first. After that, when the battery's voltage reaches the rated voltage, the IC change the $\mathrm{CC}$ mode to the constant voltage (CV) mode. The supply current decreases gradually and IC stop to supply when the current becomes below the threshold current value $\left(\mathrm{I}_{\mathrm{TH}}\right)$.

\section{How to design of electrodes which can extend freely under the certain conditions}

The proposed contact-type battery charger includes some electrodes but all kinds of design of that cannot be used for this. In this section, we will explain how to design the electrodes which can extend freely under the certain conditions.

First of all, the minimum unit and the size of electrode and distance between electrodes is shown in Fig.4.

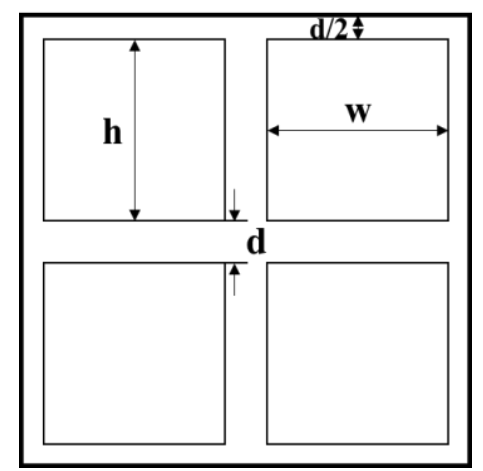

(a) For charge system

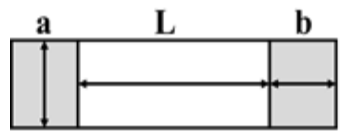

(b) For battery
Fig.4. The minimum unit size and battery size

In this case, we define 4 electrodes $(2 * 2)$ as the minimum unit. Many patterns will happen in this situation but we will explain the how to design to avoid worst case, such as a short.

When the battery's electrodes are put on a charger's electrode at the same time, a short happens. To avoid this, the valuable $L$ and $d$ have to satisfy the follow equations.

$$
\begin{gathered}
0<\mathrm{d}<\frac{\sqrt{2}}{2} \max (\mathrm{a}, \mathrm{b}) \\
\sqrt{h^{2}+w^{2}}<L<\{2 * \min (h, w)+d\}
\end{gathered}
$$

Only satisfying these, a short never happens in minimum unit. Unfortunately, minimum unit cannot connect in parallel.

Next, we will explain about basic unit. it means that the total electrodes is more than 9 , in other words, Both vertical and lateral electrodes number is more than $3(m, n \geq 3)$ in Fig.5. Basic unit can use not only single but also in parallel as shown in Fig.6. This also have certain condition like minimum unit. To avoid this, the valuable $L$ and $d$ have to satisfy the equation (1) and the follow equations. 
(1) Use single

$$
\begin{aligned}
& \sqrt{w^{2}+h^{2}}<L<\min (x, y) \\
& {\left[\begin{array}{l}
x=w * n+d *(n-1) \\
y=h * m+d *(m-1)
\end{array}\right]}
\end{aligned}
$$

In this case, $m, n>2$

(2) Use in parallel

$$
\begin{aligned}
& \sqrt{w^{2}+h^{2}}<L<\min \left(x^{\prime}, y^{\prime}\right)-2 b \\
& {\left[\begin{array}{c}
x^{\prime}=w *(n-1)+d * n \\
y^{\prime}=h *(m-1)+d * m
\end{array}\right]}
\end{aligned}
$$

\section{In this case, $\mathbf{m}, \mathbf{n}>2$}

When basic unit is used in parallel, same position's electrode in one unit connected with other same position electrode. For example, in Fig.5., same color electrode is connected in parallel so they can be considered as one electrode.

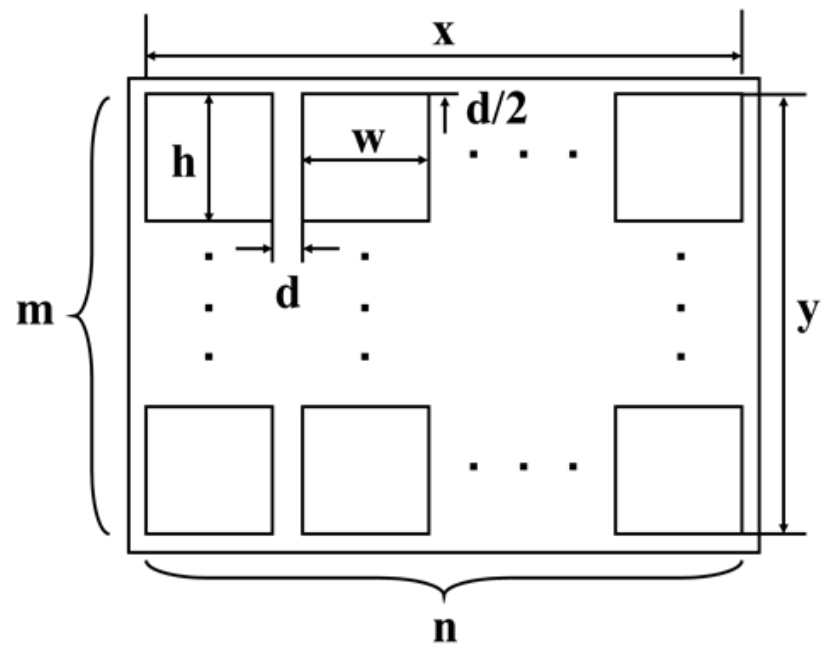

Fig.5. The figure of basic unit

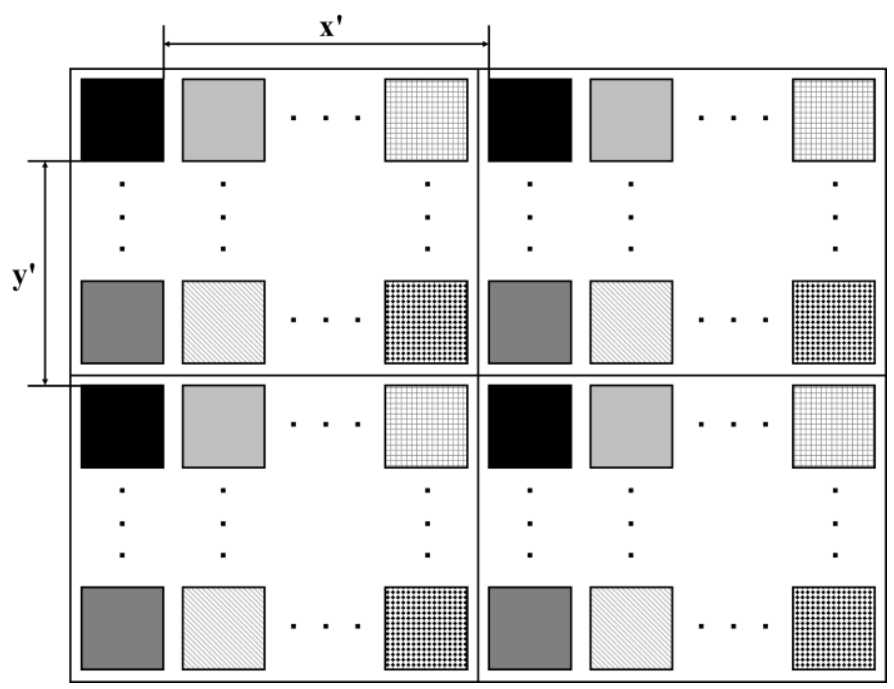

Fig.6. The figure of electrode connected in parallel

\section{Experimental result}

We used 7 segment LEDs and BCD-to-Seven segment decoder IC to check whether my program was working correctly. (7 segment LED and BCD-to-Seven segment decoder IC were used only for checking program errors.) In the program, the signal $i$ and $j$, that is the battery position and polarity, are displayed when the result of $A / D$ conversion is not zero. For instance, if the signal i is $2, \mathrm{j}$ is 4 and A/D conversion is not zero, the number " 24 " is displayed on the 7 segment LEDs such as Fig.7. Furthermore, the left digit shows the battery's positive electrode position and the right one shows the negative positon. Therefore, by watching the number, I could check whether the program is working correctly.

We experimented under the follows situations;

- Charge current $500 \mathrm{~mA}$

- Battery Li-ion battery with protect-function $186503.7 \mathrm{~V} / 2,800 \mathrm{mAh}$

As a result, the circuit of detection system worked correctly and the number of electrode which the battery is put on was displayed on the 7 segment LED module.

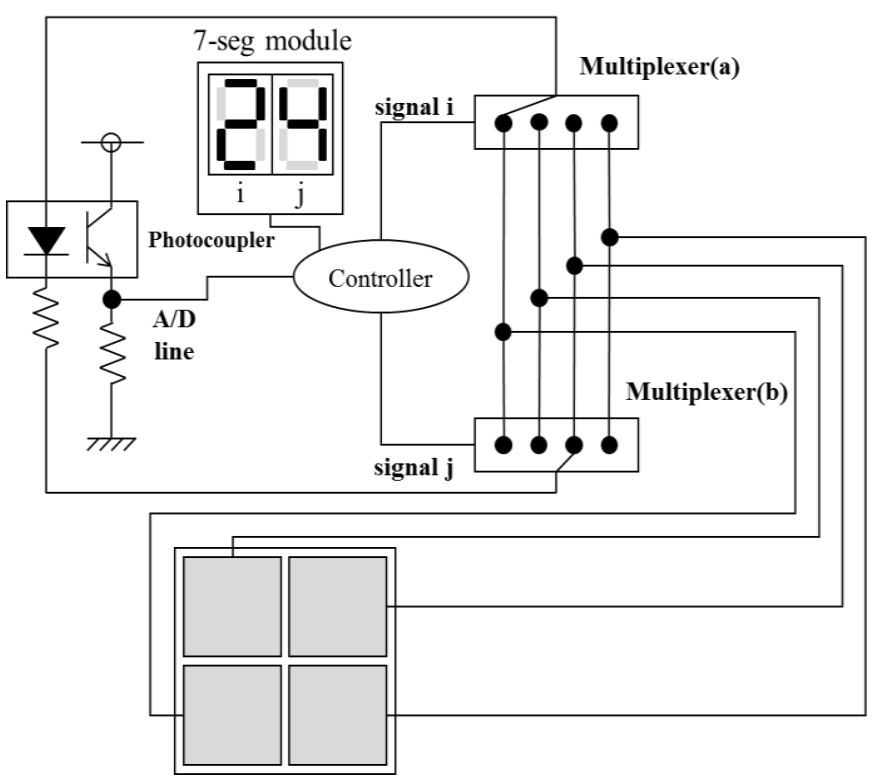

Fig.7. The schematic for experiment

\section{Conclusion}

We could show and experiment the principle of contact-type charger which can use without position control, the design of electrodes which can extend under the certain conditions.

As future work, we would like to try miniaturize the circuit scale and to develop the automatic charge 
management system with contact-type positioning free charger.

\section{References}

(1) Daisuke Tanaka, Masaharu Moritsugu, Mayu Yamanaka, Lifeng Zhang : "Development of Contact Type Positioning-free Supplier and Charger", The 2nd International Conference on Industrial Application Engineering 2014, 2014 